\title{
Thymic stromal alterations and genetic disorders of immune system
}

\author{
Claudio Pignata ${ }^{1}{ }^{*}$, Roberta D'Assante ${ }^{1}$ and Ana E. Sousa ${ }^{2}$ \\ ${ }^{1}$ Department of Translational Medical Sciences, Federico II University, Naples, Italy \\ 2 Instituto de Medicina Molecular, Faculdade de Medicina, Universidade de Lisboa, Lisboa, Portugal \\ *Correspondence: pignata@unina.it
}

Edited and reviewed by:

Ellis L. Reinherz, Dana-Farber Cancer Institute, USA

Keywords: severe combined immunodeficiency, FOXN1, central tolerance, medullary thymic epithelial cells, DiGeorge syndrome, rag defects, APECED

In this specialty section of the journal, we host a topic focused on thymic stromal alterations and genetic disorders of immune system. The thymus is a specialized organ of the immune system where, through stage-specific differentiation of hematopoietic progenitor cells, fully mature and self-tolerant $\mathrm{T}$ cells origin. The process is strictly dependent on the link between the thymic stromal cells (TSCs), which allow the selection of a functional and self-tolerant $\mathrm{T}$-cell repertoire, and the thymus tridimensional architecture. Indeed, the interaction between the developing thymocytes and the stromal cells is crucial for the development of both T cells and TSCs $(1,2)$. In both human and mice, the primordial thymic epithelial cells (TECs) are yet unable to fully support the T-cell development and only after the transcriptional activation of the Forkhead-box $n 1$ (FOXN1) gene, this essential function is acquired. Most of the information concerning the T-cell development came out from studies on mice carrying null mutation in FOXN1 gene. In humans, as detailed in the Romano et al. review, the Nude/SCID phenotype is characterized by congenital alopecia of the scalp, eyebrows, and eyelashes, nail dystrophy, and a severe T-cell immunodeficiency, inherited as an autosomal recessive disorder (3). As extensively approached in the Villa et al. review, the intercellular cross-talk is also essential to support the maturation of Foxp3C natural regulatory $\mathrm{T}$ cells. In Omenn syndrome (OS), caused by hypomorphic Rag alterations, an infiltration of peripheral tissues by activated $\mathrm{T}$ cells and immune dysregulation have been found (4). The authors discuss on abnormalities of thymic microenvironment in OS with a special focus on the defective maturation of TECs, and impairment of central tolerance.

The commonest association of thymic stromal deficiency resulting in T-cell immunodeficiency is the DiGeorge syndrome (DGS), discussed in the Davies review. In this syndrome, however, the immunological impairment is highly variable, ranging from normal to a severe immune defect in rare individuals, thus suggesting that partial thymic hypoplasia may occur or that extrathymic sites of differentiation play a role in the process (5). The difference in the immunological defects between DGS and the Nude/SCID phenotypes implies that FOXN1 controlled genes are mandatory for a fully mature T-cell development process rather than the integrity of the thymus itself.

It is known that autoimmune regulator (AIRE) gene plays a central role in the induction of central tolerance, and different mechanisms of action have been hypothesized for this process. According to the most reliable theory, AIRE directly induces the production of tissue-specific antigens (TSA) (6). However, recent evidence suggests that another mechanism for negative selection of self-reactive thymocytes may be due to AIRE-induced differentiation of medullary TECs, and regulation of the expression of intrathymic chemokines directed to antigens presenting cells (APCs), such as thymocytes and dendritic cells (7). In their reviews, Laan and Peterson and Kisand et al. give an overview on what is known about the different mechanisms through which AIRE induces central tolerance.

The process aimed at the elimination of potential self-reactive T cells in the thymus is crucial for preventing the onset of autoimmune diseases. As discussed in the Akiyama et al. paper, medullary epithelial cells play a central role in the process through the regulation of gene expression, and, in particular, of those genes encoding for the TNF family cytokines, RANK ligand, CD40 ligand, and lymphotoxin. These genes promote the differentiation of AIRE- and TSA-expressing mTECs (8).

The mechanism by which a single AIRE gene can influence the transcription of such a large number of TSA within mTECs has been discussed in the Matsumoto et al. paper. Two models have been proposed. The first one implies a direct transcriptional control of AIRE on TSA, while the second one is based on the role of AIRE on the maturation program of mTECs (9).

The clinical and immunological phenotype of patients affected with autoimmune polyendocrinopathy ectodermal dystrophy (APECED), as reviewed by Petteri Arstila and Jarva, is characterized by multiple endocrine deficiencies, the most common manifestations being hypoparathyroidism, Addison's disease, hypogonadism, and secondary amenorrhea, usually associated with the presence of autoantibodies toward the target tissues (10). However, the phenotype and, therefore, the underlying pathogenic mechanism, are even more complex, in that Chronic Mucocutaneous Candidiasis is also a prominent part of the disease. This clinical entity is related to abnormalities in the Th17-related cytokines, which are mostly involved in immune defenses against Candida (11). Finally, high titers of neutralizing autoantibodies against type I interferons, which have been shown to downregulate the expression of interferon-controlled genes, have been documented (12).

In this Research Topic, De Martino et al. focus their attention on the complexity of the APECED phenotype in that a wide variability of the clinical expression, in the presence of the same genotype alteration, has been found (13). They suggest that additional 
mechanisms, in addition to AIRE function, are involved in the pathogenesis of the disease. This might be helpful to understand not only the molecular basis of APECED but will also help improve diagnosis, management, and therapeutic strategies to treat this complex disease.

\section{REFERENCES}

1. Anderson G, Jenkinson EJ. Lymphostromal interactions in thymic development and function. Nat Rev Immunol (2001) 1:31-40. doi:10.1038/35095500

2. Romano R, Palamaro L, Fusco A, Giardino G, Gallo V, Del Vecchio L, et al. FOXN1: a master regulator gene of thymic epithelial development program. Front Immunol (2013) 4:187. doi:10.3389/fimmu.2013.00187

3. Pignata C, Fiore M, Guzzetta V, Castaldo A, Sebastio G, Porta F, et al. Congenital alopecia and nail dystrophy associated with severe functional T-cell immunodeficiency in two sibs. Am J Med Genet (1996) 65:167-70. doi:10.1002/(SICI) 1096-8628(19961016)65:2<167::AID-AJMG17>3.0.CO;2-O

4. Corneo B, Moshous D, Güngör T, Wulffraat N, Philippet P, Le Deist FL, et al. Identical mutations in RAG1 or RAG2 genes leading to defective $\mathrm{V}(\mathrm{D}) \mathrm{J}$ recombinase activity can cause either T-B-severe combined immune deficiency or Omenn syndrome. Blood (2001) 97:2772-6. doi:10.1182/blood.V97.9.2772

5. Davies EG. Immunodeficiency in DiGeorge syndrome and options for treating cases with complete athymia. Front Immunol (2013) 4:322. doi:10.3389/fimmu. 2013.00322

6. Anderson MS, Veneziani ES, Klein L, Chen Z, Berzins SP, Turley SJ, et al. Projection of an immunological self shadow within the thymus by the aire protein. Science (2002) 298:1395-401. doi:10.1126/science.1075958

7. Derbinski J, Kyewski B. How thymic antigen presenting cells sample the body's self-antigens. Curr Opin Immunol (2010) 22:592-600. doi:10.1016/j.coi.2010. 08.003

8. Akiyama T, Shinzawa M, Akiyama N. TNF receptor family signaling in the development and functions of medullary thymic epithelial cells. Front Immunol (2012) 3:278. doi:10.3389/fimmu.2012.00278

9. Matsumoto M, Nishikawa Y, Nishijima H, Morimoto J, Matsumoto M, Mouri Y. Which model better fits the role of aire in the establishment of self-tolerance: the transcription model or the maturation model? Front Immunol (2013) 4:210. doi:10.3389/fimmu.2013.00210

10. Kisand K, Peterson P. Autoimmune polyendocrinopathy candidiasis ectodermal dystrophy: known and novel aspects of the syndrome. Ann N Y Acad Sci (2011) 1246:77-91. doi:10.1111/j.1749-6632.2011.06308.x

11. Kisand K, Boe Wolff AS, Podkrajsek KT, Tserel L, Link M, Kisand KV, et al. Chronic mucocutaneous candidiasis in APECED or thymoma patients correlates with autoimmunity to Th17-associated cytokines. J Exp Med (2010) 207:299-308. doi:10.1084/jem.20091669

12. Meloni A, Furcas M, Cetani F, Marcocci C, Falorni A, Perniola R, et al. Autoantibodies against type I interferons as an additional diagnostic criterion for autoimmune polyendocrine syndrome type I. J Clin Endocrinol Metab (2008) 93:4389-97. doi:10.1210/jc.2008-0935

13. Capalbo D, Fusco A, Aloj G, Improda N, Vitiello L, Dianzani U, et al. High intrafamilial variability in autoimmune polyendocrinopathy-candidiasisectodermal dystrophy: a case study. J Endocrinol Invest (2012) 35:77-81. doi: $10.3275 / 8055$

Conflict of Interest Statement: The authors declare that the research was conducted in the absence of any commercial or financial relationships that could be construed as a potential conflict of interest.

Received: 16 January 2015; accepted: 11 February 2015; published online: 25 February 2015.

Citation: Pignata $C, D^{\prime}$ Assante $R$ and Sousa AE (2015) Thymic stromal alterations and genetic disorders of immune system. Front. Immunol. 6:81. doi: 10.3389/fimmu.2015.00081

This article was submitted to T Cell Biology, a section of the journal Frontiers in Immunology.

Copyright (C) 2015 Pignata, D'Assante and Sousa. This is an open-access article distributed under the terms of the Creative Commons Attribution License (CC BY). The use, distribution or reproduction in other forums is permitted, provided the original author(s) or licensor are credited and that the original publication in this journal is cited, in accordance with accepted academic practice. No use, distribution or reproduction is permitted which does not comply with these terms. 